\title{
Magnetic fluxes of massive stars: statistics and evolution
}

\author{
Alexander F. Kholtygin ${ }^{1}$, Sergei N. Fabrika ${ }^{2}$, Natalia A. Drake ${ }^{1}$ and \\ Andrei P. Igoshev ${ }^{1}$ \\ ${ }^{1}$ Astronomical Instituut, Saint-Petersburg University, \\ 198504, ul Shaxmatova, Petrodvoretz, Rissia \\ email: afkholtygin@gmail.com \\ ${ }^{2}$ Special Astrophysical Observatoty, Nizhnii Arxyz, Russia
}

\begin{abstract}
The statistical properties of magnetic fields and magnetic fluxes of OB stars were investigated. The mean magnetic fluxes of massive OB stars appear to be 3 order larger than those for neutron stars.
\end{abstract}

Keywords. stars: early-type, stars: magnetic fields, stars: neutron

\section{Magnetic Fields}

We collect the recent measurements of the magnetic fields of OB stars from the catalogue of Bychkov et al. (2009) and the newest data from the literature. Basing on these data we have investigated the statistical properties of an ensemble of magnetic fields of OB stars. As a statistical measure of the stellar magnetic field we used the rms longitudinal magnetic field $\mathcal{B}$. The mean magnetic fields for OB stars averaged over the spectral subclasses are plotted in Fig. 1 (left panel). There is an unexpectedly large jump between $\mathrm{O}$ and $\mathrm{B}$ star mean fields.

We have calculated the normalized differential magnetic field function $(\mathrm{MFF}) F(\mathcal{B})$ for OB stars, which is determined accordingly Kholtygin et al. (2010a) in such a way that a value of $F(\mathcal{B}) d \mathcal{B}$ indicates the probability that a $r m s$ field $\mathcal{B}$ is in an interval $(\mathcal{B}, \mathcal{B}+d \mathcal{B})$ (right panel in Fig. 1). We found that the MFF for $\mathcal{B}>400 \mathrm{G}$ can be approximated by a power function: $F(B)=0.33(B / 1 k G)^{-1.82}$. For smaller values of $\mathcal{B}$ the values of the MFF are lower than those obtained from the power fit by more than an order of magnitude. We suppose that this deviation can be connected with observational bias.

To study this effect we suppose that the real MFF is described by the power law for all values of $\mathcal{B}<M_{\min }=20 \mathrm{G}$. We calculated with our Monte-Carlo code the field detection probability $P(n, \sigma, \mathcal{B})$ for a star with the $r m s$ field $\mathcal{B}$, a fixed error $\sigma$ of field measurement and $n$ polarimetric observations (for details see Kholtygin et al. 2010a). It appears that a value of $P(n, \sigma, \mathcal{B})$ weakly depends on the $n$ for $n \geqslant 3$. We use this probability to restore the quasi-observed MFF which could be obtained in a case of observation of all stars using the polarimeter with a fixed value of $\sigma$. The restored values were calculated by Kholtygin et al. (2010b). It appeared that the observed MFF cannot be made to agree with the restored one for any possible values of $\sigma$. We connect this fact with an additional factor of decreasing MFF, probably with destroying the weak fields by field instabilities, as proposed by Aurière et al. (2007). 

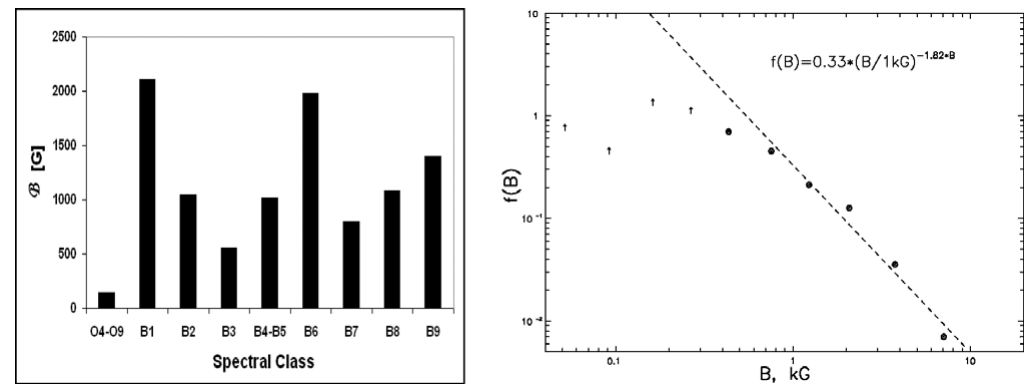

Figure 1. Left Panel: Mean Magnetic Fields of OB stars for different spectral subclasses. Right Panel: MFF for OB stars obtained on the measured magnetic fields for $\mathcal{B} \geqslant 400 \mathrm{G}$ (points) and for $\mathcal{B}<400 \mathrm{G}$ (arrows). Power fit for MFF is shown with a dashed line.
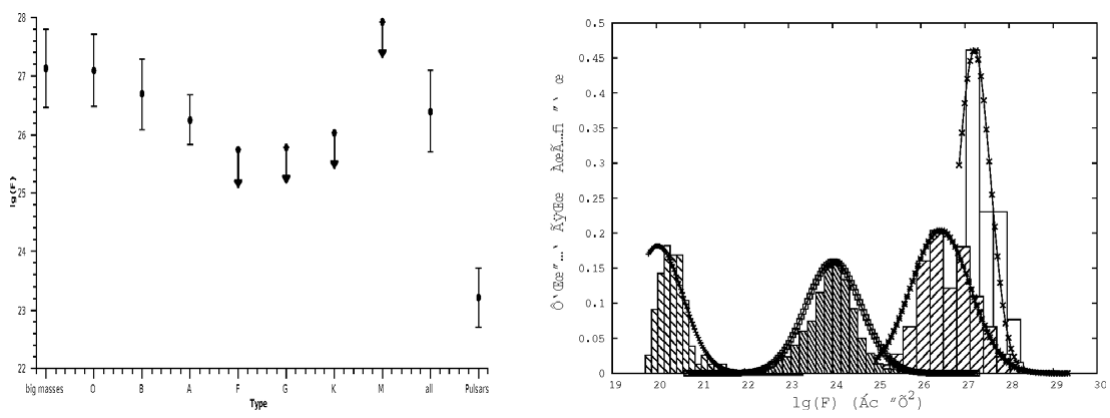

Figure 2. Left Panel: Mean magnetic fluxes for normal stars, massive stars and pulsars vs. their spectral class. Right Panel: Distributions of the magnetic fluxes for millesecond pulsars, normal pulsars, OB stars and magnetars (from left to right) and their log.-normal fit.

\section{Magnetic Fluxes}

For all studied stars we have estimated the magnetic fluxes using a relation $\mathcal{F}=4 \pi R_{*}^{2} \mathcal{B}$, where $R_{*}$ is a photospheric radius of the star. We established that the magnetic fluxes for stars of all spectral classes have a log-normal distribution. Mean magnetic fluxes as a function of spectral class are given in Fig. 2 (left panel). For stars of F and later spectral classes only upper limits of magnetic fluxes can be found (arrows). We also calculated the magnetic fluxes for neutron stars (NSs) using their magnetic fields from ATNF Pulsar Catalogue (http://www.atnf.csiro.au/research/pulsar/psrcat) and McGill SGR/AXP Online Catalog (http://www.physics.mcgill.ca/ pulsar/magnetar/main.html) and accepting for all NS the typical radius $R_{*}=10 \mathrm{~km}$. Mean magnetic fluxes for most NSs appear to be up to 3 orders of magnitude lower than those for massive OB stars. This means that massive stars lose the lion's share of their magnetic flux. The magnetic flux distributions for NSs and OB stars are plotted in Fig. 2 (right panel). The distribution of magnetic fluxes for magnetars is narrow and it is shifted by 0.4 dex relative to that of OB stars.

\section{References}

Bychkov, V. D., Bychkova, L. V., \& Madej, J. 2009, MNRAS, 394, 1338

Aurière, M., Wade, G. A., Silvester, J., Lignières, F. et al. 2007, A\&A, 475, 1053

Kholtygin, A. F., Fabrika, S. N., Drake, N. A., Bychkov, V. D. et al. 2010a, Astron. Lett., 36, 370

Kholtygin, A. F., Fabrika, S. N., Drake, N. A., Bychkov, V. D. et al. 2010b, Kinematics and Physics of Celestial Bodies, 26, 181 